REVISTA ANDALUZA DE ANTROPOLOGÍA.

NÚMERO 7: GITANOS/ROMA:AUTO-PRODUCCIÓNCULTURAL Y CONSTRUCCIÓN HISTÓRICO-POLÍTICA SEPTIEMBRE DE 2014

ISSN 2174-6796

[pp. 44-61]

http://dx.doi.org/10.12795/RAA.2014.i07.03

Fecha de Recepción: 18-03-2014

Fecha de Aceptación: 09-06-2014

\title{
LA GENEROSIDAD COMO ARTE. IDENTIDAD, FRATERNIDAD Y JERARQUÍA ENTRE LOS GITANOS DE DOS PUEBLOS ANDALUCES
}

\author{
Nathalie Manrique \\ École des Hautes Études en Sciencies Sociales
}

\section{Resumen.}

Para los Gitanos de Morote y San Juan (Andalucía), no tiene sentido acumular bienes. De hecho, el don ordena en primer lugar individuos y grupos en categorías jerárquicas "naturales" (heredadas al nacer) donde el donante, percibido como "naturalmente" generoso, está considerado como superior al recipiente. Aquí la reciprocidad es impensable. De esta manera, los seres humanos son superiores a los animales, los Gitanos a los Payos, hombres y mujeres, etc. Por lo tanto, la acumulación de dinero, podría poner en peligro la jerarquía, y entonces la gitanidad de cada uno.

Dentro de cada categoría "natural”, los estatutos se reajustan periódicamente por medio de transacciones recíprocas (contradon). Esto permite una especie de reequilibrio de la riqueza (simbólica o material) que favorece la igualdad de estatus entre pares.

Esta falta de capitalización se puede también observar en la evitación de la transmisión de los bienes después de la muerte de su posesor: todo lo que recuerda a los muertos se “olvida", se quema o se entierra. Por lo tanto, el estatus y la riqueza de los Gitanos no se pueden heredar: en este contexto, la jerarquía gitana en estos pueblos tiene pies firmes.

Palabras clave.

Gitanos, don, riqueza, jerarquía, paridad, muerte. 


\section{Abstract.}

For the Gypsies of San Juan y Morote (Andalusia), there is no point in accumulating goods. In fact, gift orders in a first place individuals and groups in "natural" hierarchical categories (inherited at birth) where the donor, perceived as "naturally" generous, is considered to be superior to the recipient. Thus, reciprocity is unthinkable. In this case humans are superior to animals, Payos to Gypsies, men to women and so on. Therefore, the accumulation of money, could endanger the hierarchy and then each gypsyness.

Within each "natural" category, the statutes is periodically reset through reciprocal transactions (counter-gift). This allows a kind of rebalancing of (symbolic or material) wealth that favors equality of status among peers.

This lack of capitalization can also be observed in the avoidance of the transfer of property after the death of its possessor: everything which reminds the dead is "forgotten", burned or buried. Therefore, Gypsies' status and wealth cannot be inherited: in this context, gypsy hierarchy has strong feet in these towns.

\section{Keywords.}

Gypsies, gift, wealth, hierarchy, parity, death.

Morote y San Juan ${ }^{1}$, dos pueblos con respectivamente unos 9.000 y 4.600 habitantes, de los cuales aproximadamente unos 800 y casi 450 son Gitanos, están ubicados en los montes orientales granadinos. Antaño, los Gitanos procedentes de estas dos pequeñas villas practicaban mayormente los oficios relacionados con los caballos, burros y mulos: eran chalanes o esquiladores. En la actualidad, esta población, al igual que la mayoría de los españoles gitanos, es sedentaria ${ }^{2}$, aunque su principal actividad económica como peones del campo les obliga a viajar con frecuencia. Cuando están en casa, complementan sus ingresos con la venta de cestas de mimbre hechas a mano, ya sea puerta a puerta o bajo el pedido de sus vecinos no gitanos (payos o castellanos).

Los Gitanos nunca cupieron fácilmente en la sociedad española fuertemente jerarquizada donde, como en otras partes de Europa ${ }^{3}$, la ideología dominante legitimaba una jerarquía social basada principalmente en los lazos territoriales, la descendencia y la acumulación de riqueza. En cambio, los Gitanos españoles establecieron su estatus y valores de acuerdo a cualidades opuestas: la movilidad y, lo más importante, el rechazo de la acumulación de

1. Correcciones de texto de David Lagunas. Para preservar el anonimato de mis informantes, he cambiado el nombre de estos dos pueblos.

2. Leblon (1985).

3. Diversos estudios históricos y antropológicos de Europa destacan la estrecha relación entre la identidad y el patrimonio a través de las prácticas matrimoniales (por ejemplo, Bestard Camps 1986; Bourdieu 1980; Segalen 1985; Zonabend 1980). 
bienes y de la transmisión de los símbolos del poder y de la riqueza. Incluso, practicaban y siguen practicando la destrucción de los bienes materiales y simbólicos que pertenecían a un difunto. Todo esto implica bien la circulación o destrucción de bienes. Esto primero implica donantes y receptores de los productos en cuestión. Para los Gitanos de Morote y San Juan, el don clasifica y jerarquiza individuos y grupos en función de la superioridad presupuesta de los donantes.

En primer lugar, el don ${ }^{4}$ clasifica los individuos y grupos en categorías heredadas, entonces consideradas como "naturales", que están jerárquicamente relacionadas según dos principios fundamentales: la presupuesta intensidad de los intercambios entre los grupos y individuos (que son bien donantes o receptores) y la dirección de sus transferencias. Aquí, el don no implica que se devuelvan los bienes y además esto no es concebible en este contexto.

Dentro de cada categoría, se supone que debe reinar la igualdad (donantes pueden llegar a ser receptores y viceversa). Aquí, la obligación de devolver lo adquirido -que Mauss, 1959 llama "contradon (contre-don)"-, incluso si el acervo no es deseado por el receptor potencial (es que la toma incluye una posición de inferioridad), y precisamente por ello, implica una dinámica intensa de intercambio dentro de la categoría.

Las prácticas funerarias de los Gitanos de Morote y San Juan, a través de la destrucción u "olvido" (es un olvido formal pero no real: hasta que desaparezcan igualmente las personas que conocieron el/la difunto/a) de todo lo que pertenecía a la persona fallecida (bienes personales, apodos, etc.), ponen de relieve la falta de herencia de riquezas y del estatus, y favorecen este ideal de igualdad: entre los Gitanos: los bienes no son destinados a ser acumulado, sino que son una herramienta para que las personas realicen su capacidad de dar, y de esta manera para lograr un estatus mayor dentro de una u otra categoría.

\section{Ser generoso/a Por nacimiento}

Según Robert Hertz (1990 [1909]), “Toda jerarquía social se pretende fundada sobre la naturaleza de las cosas, razón por la que se le atribuye vigencia eterna, escapando al devenir y a las disputas de los innovadores." Para los gitanos de Morote y San Juan, algunas categorías están pensadas como "naturales" porque distinguen los individuos y grupos en función de criterios adquiridos por nacimiento (el hecho de ser un no humano, un ser humano, un/a Gitano/a, un/a Payo/a, un hombre, una mujer, etc.). Estos logros innatos legitiman y son legitimados por el proceso del don: en la cosmología gitana,

\footnotetext{
4. Este articulo trata de construir un modelo dentro del cual los discursos y comportamientos observados adquieren un sentido global. En ningún momento, postulo que todos los Gitanos españoles actúan según este modelo ( $\tan$ siquiera los de Morote y San Juan). Pero sí que hace parte de la referencia ideal de comportamiento. El que no lo sigue es considerado como "apayao" o como un Gitano poco respetable. Y su castigo puede ser que ya no encuentre pareja para casarse en su pueblo. Además, este modelo no se fijó una vez para siempre pero se modifica con el tiempo..
} 
los grupos y los individuos se clasifican de acuerdo con un mecanismo que, en algunos aspectos, es muy similar al proceso general del don desarrollado por Mauss (1925) en que el donante es superior al recipiente, cuando éste no está en condiciones, por razones socio-económicas o ideológicas, de devolver lo que recibió: "el don no devuelto hace que sea la persona que lo aceptó aún más inferior, sobre todo cuando lo aceptó sin pensar en devolverlo" (Mauss 1925: 160) ${ }^{5}$. En suma, el acto por el cual un individuo da un bien simbólico o material a otro coloca automáticamente este primero a un estatus superior. Por lo tanto, todo el mundo se siente obligado a devolver lo dado. De hecho, otro don hecho al donante original reequilibra esta situación. Esto es lo que Mauss (1925) llama "contra-don" y lo que Lévi-Strauss (1949) describe con la noción de "principio de reciprocidad (príncipe de réciprocité)".

Esta obligación de devolver, fundamental para la teoría de Mauss, no es aplicable a los intercambios entre personas de distintas categorías dentro de la "clasificación natural" de los seres, ya que se supone que esta clasificación debe ser permanente y cerrada: los cambios de su orden jerárquico son imposibles. Esta clasificación se basa en primer lugar en la generosidad (es decir, en la inclinación a dar y no a recibir) que se supone que todos poseen, aunque con distinta intensidad. De este modo, los seres humanos son superiores a los animales, los hombres a las mujeres, y así sucesivamente. Sus estatutos se basan en la intensidad de la propensión presupuesta de cada uno para dar, en sus palabras, para ser "generoso". De hecho, los Gitanos valoran mucho la generosidad ${ }^{6}$ que distingue los grupos e individuos entre ellos.

Así, para los Gitanos, la organización social parece estar estructurada de acuerdo con lo que llamo "súper-categorías" y "categorías" que enlazan a cada individuo de "manera natural" (los individuos entran en una súper-categoría o categoría por su "naturaleza", es decir, por nacimiento o paso del tiempo). Las "súper-categorías" son reconstrucciones lógicas a partir de mis propios datos tomados durante mi trabajo de campo. Aunque no se utilizaron de forma explícita por los Gitanos de estos dos pueblos, noté en sus palabras y hábitos estas distinciones. Por ejemplo, según ellos, los Gitanos de otras aldeas son "todos salvajes", ya que se supone que todos son drogadictos o ladrones, o no Gitanos de verdad (dicen que son apayaos, es decir, que su forma de ser está ahora más cerca de la de los Payos). Por lo tanto, los Gitanos locales no tienen ninguna relación con los

5. 'Le don non rendu rend encore inférieur celui l'a qui accepté, surtout quand il est reçu sans esprit de retour.'

6. La valorización de la generosidad se hizo más explícita cuando mis informantes evaluaban las cortesías de los demás cuando me recibían en sus casas. Sin que se exprese directamente, detecté un sentido de desprecio dirigido a aquellos que habían demostrado su carácter asocial hacia la extranjera que era al principio de mi estancia en estos pueblos. Tal comportamiento se equiparaba con su poca humanidad ("son unos salvajes").

7. Dicen: "es natural" o "es asi". 
Gitanos que "no son como nosotros". Las "categorías" son entidades funcionales y sus fronteras se construyen a través de la circulación de bienes: los intercambios tienen lugar regularmente dentro de estas categorías y con Gitanos locales de otras categorías. Pero en este último caso, los movimientos de mercancías se niegan por completo por parte de las categorías superiores. Esto es un buen indicio que precisa los límites y rango de cada categoría (Figura1).

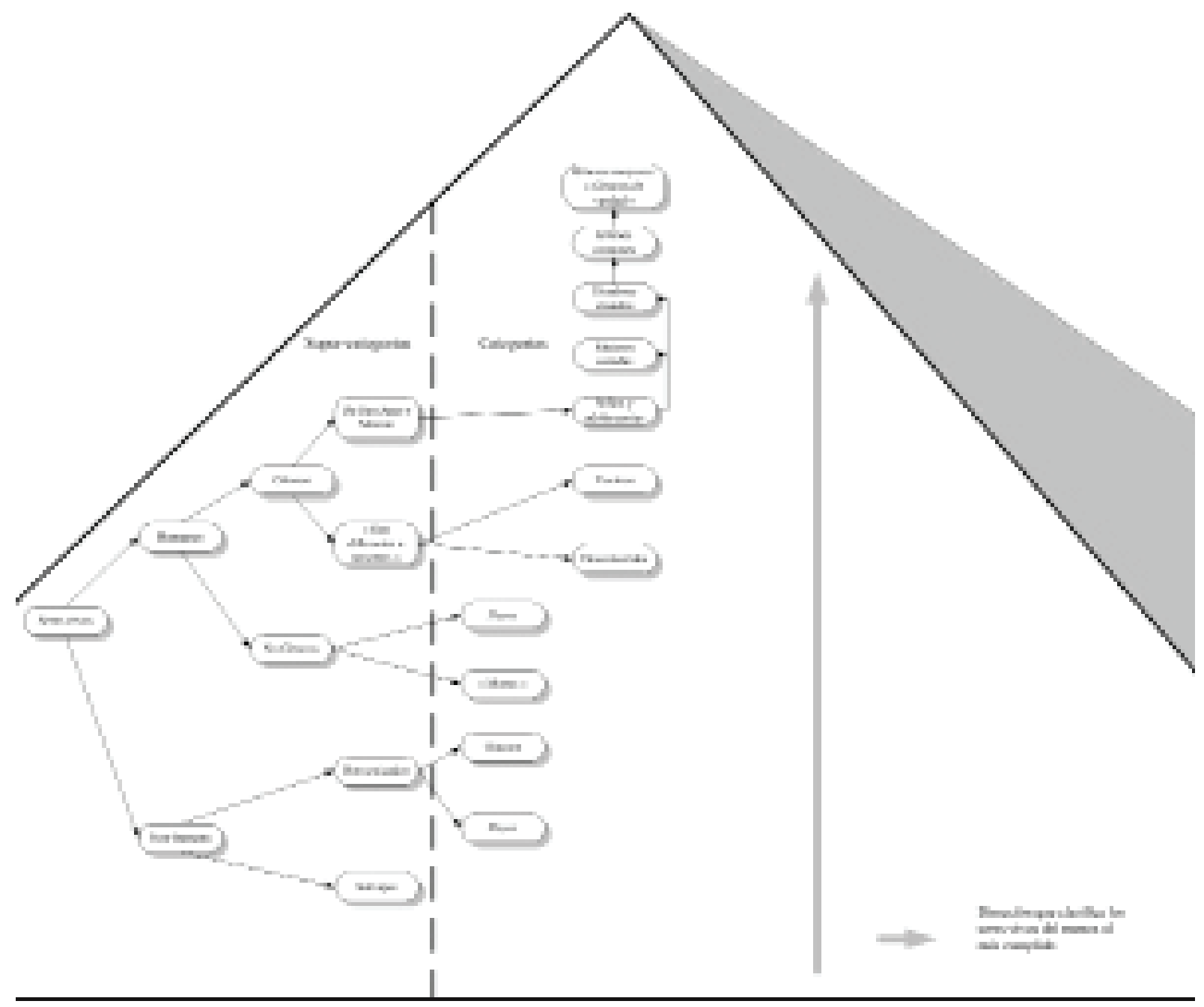

Figura 1: Clasificación "natural"

8. Ninguno de los bienes se conservan. Este es también el caso con el dinero (ver también Stewart, 1994 para el caso de los Gitanos húngaros) que se presta, a veces incluso a Payos, con mayores tasas de interés. El interés se usará generalmente para otros préstamos. 
Para los gitanos, las categorías menos inclusivas se acercan más al ideal gitano: la imagen se parece bastante a la de la destilación: en el nivel más "refinado", casi divino, se encuentra la identidad completa del Gitano. Esta "quintaesencia" gitana se refleja mejor en la categoría suprema del don, es decir, en la categoría donde se encuentran los donantes por excelencia, la que subordina todas los demás sin integrarlas: está constituida por los hombres gitanos de mayor edad. Estos hombres son, en suma, los "verdaderos" Gitanos -los Gitanos dicen que son los "Gitanos de verdad". De hecho, la identidad gitana parece cuantificarse de acuerdo con esta propensión presupuesta de cada uno para dar: el más generoso es, desde el punto de vista de gitano, el más Gitano (de hecho, el que más se acerca al ser humano cumplido: ver la Figura 1).

Me di cuenta de este carácter cuantificable de la gitanidad durante una conversación con Antonio, un Gitano de unos cuarenta años de edad y padre de siete hijos de corta edad. Un día le aconseje de alistarse con la asociación gitana local, a fin de recibir ayuda alimentaria de la Cruz Roja. De repente, pareció ofendido y dijo: "Yo soy bastante gitano como para no tener que pedirle ayuda a otro [es decir, a otro Gitano, en referencia al presidente de la asociación]. Al parecer, al aceptar dicha asistencia podría limitar o reducir su hombría gitana (es decir, su grado de gitanidad basado en su edad, sexo, pertenencia a la red local, etc.).

Parece que solo los hombres tienen la capacidad de alcanzar este estatus máximo: con la madurez se supone que han demostrado ya su generosidad y llegan a ser, en cierto modo, donantes perennes. De hecho, esta generosidad se basa esencialmente en las alianzas matrimoniales, las cuales, para los Gitanos de Morote y San Juan, es el punto de partida más importante de varios lazos que activarán la circulación de bienes entre pares. Por lo tanto, legitima el estado de los ancianos, ya que se supone que todos los hombres de cierta edad que casaron varios de sus hijos, y por lo tanto que han participado activamente en una serie de dones (principalmente por la procreación ${ }^{9}$, sino también por celebrar banquetes nupciales - cuya suntuosidad es a menudo una cuestión de discusiones mucho tiempo después-). En realidad, los ancianos representan un nivel mayor de "gitanidad" y son los que mejor personifican el estatus ideal dentro de la comunidad gitana. Por lo tanto, ampliamente respetados, son ellos los que pueden llevar el sombrero o la gorra, el bigote y el garrote (o vara) sin riesgo de ser blancos de burlas. Recuerdo el caso de Manuel, un robusto hombre de unos cuarenta años, que sufrió muchas críticas como presidente de la asociación gitana local mencionada anteriormente. Un día, decidió llevar el sombrero, el bigote y la vara generalmente reservados a los hombres gitanos

9. Ver Manrique (2013). 
mayores. Después de someterse a una increíble cantidad de abucheos y burlas ${ }^{10}$, se quitó rápidamente todas estas marcas de prestigio.

Para ser un "donante absoluto", que, como hemos visto, depende del ciclo de vida, otros deben ser, "por naturaleza", sus recipientes (o donatario) perpetuos. Este es el caso, por ejemplo, con los Payos o Castellanos. José me relató que los ancianos contaban que los primeros Gitanos surgieron, con las herramientas para esquilar en mano, de los excrementos de un caballo cuando Dios administró una patada a un caballo, mientras que al mismo tiempo echaba una flatulencia ${ }^{11}$. Por otro lado, Castellanos serían los descendientes de un racimo de uva. José concluyó con: "Somos de castas diferentes"12. Esta historia alude a las respectivas actividades económicas que se espera que los gitanos y los payos ejerciten: por un lado, la agricultura y el sedentarismo es decir los que recibe su riqueza de los productos de la tierra que explotan que también pueden acumular. Mientras tanto, los Gitanos están aquí asociados con las profesiones relacionadas con los caballos (compra y venta) y el nomadismo, por lo que es difícil acumular cualquier riqueza ${ }^{13}$.

10. Los jóvenes gitanos de estos dos pueblos están acostumbrados a componer y cantar (a veces con una guitarra) canciones con palabras que glorifican o, a veces critican, aspectos de la conducta de otro. Cuando son críticas, agradan mucho a los oyentes que se ríen a carcajadas. En realidad, estas no son transgresoras ya que las víctimas son las que ya se denigran a causa de la reputación de su familia (por el comportamiento considerado como inaceptable de uno o varios de sus familiares) o de su propio comportamiento en un pasado más o menos reciente.

11. Al principio pensé que esta historia era una broma (a algunos Gitanos les gusta bromear con los extranjeros con fábulas narradas como si fueran hechos históricos). Pero varios hombres gitanos de confianza me contaron la misma historia. De vuelta en París, leí en Courthiade (2004) que historias muy similares circulan entre los grupos gitanos de los Cárpatos. En realidad, esta historia parece ser, en Morote y San Juan, una reinterpretación del acto de procreación con la semilla masculina como única contribución activa al proceso de fecundación (ver Manrique 2004, 2009).

12. Parece que José quería expresar una distinción "natural" sin usar la palabra peyorativa "raza", que está, sin embargo, comúnmente utilizada por otros grupos gitanos españoles (ver Ardévol 1986; Gay y Blasco 1999; Lagunas 2000a; San Román 1976).

13. De acuerdo con los archivos parroquiales de Morote y San Juan, los Gitanos de estas dos pequeñas ciudades han ejercido tradicionalmente las profesiones directamente relacionadas con los caballos (chalanes y esquiladores). En esta remota y montañosa región, donde a menudo las distancias entre las ciudades y pueblos superan con frecuencia varias decenas de kilómetros con rutas y caminos muy empinados, el movimiento de personas, bienes y material agrícola se hacía generalmente gracias a las bestias de carga. Además, históricamente, la economía local dependía principalmente de los labores agrícolas. La compra y venta de las bestias era entonces de suma importancia: permitían a los agricultores locales trabajar sus tierras, eran utilizadas para el transporte de productos agrícolas, para desplazarse más fácilmente, etc. Los Gitanos de estos pueblos, al no tener tierra (en el pasado, las diferentes leyes reales, pragmáticas, les impedía ser dueño de cualquier terreno y el ejercicio de las profesiones relacionadas con el mismo; ver Leblon 1985; Sánchez Ortega, 1977), se pusieron rápidamente a tener ocupaciones comerciales que les permitían satisfacer sus necesidades y las de sus familias. También practicaban la cestería que era muy útil diariamente para las familias campesinas (cestas grandes para verduras o ropa, canastas, etc.). 


\section{EL TRABAJO COMO VALOR FUNDAMENTAL}

Para los Gitanos, a diferencia de los Payos, las actividades de subsistencia no hacen hincapié en la transformación de la naturaleza a través del trabajo (es decir en la producción), sino más bien en el control de las relaciones sociales, sobre todo en los intercambios. De hecho, ser muy activo es altamente valorado entre los Gitanos de estos pueblos. Esto se expresa en su discurso con la palabra castellana "trabajo" o "trabajar" y también por el verbo "hacer", acompañado de un calificativo o una perífrasis que subraya enfáticamente el esfuerzo. El trabajo se refiere en general a todas las formas de actividad que son difíciles de lograr, ya sea debido a la complejidad, la fuerza física o la resistencia requerida. Recuerdo una conversación entre vecinos, cuando Rubén, un niño con once años, que deseando presentar una imagen positiva de su abuelo materno, insistió en su increíble capacidad por el trabajo, a pesar de tener una de sus piernas amputadas.

Esta visión de la acción está en acuerdo con las representaciones gitanas del acto sexual: se piensa que es llevado a cabo por hombres que "siembran la semilla en el cuerpo de las mujeres", y es así percibido principalmente como un don de esperma ${ }^{14}$ (bien la intención sea procrear o no $)^{15}$. El acto sexual es percibido como una sucesión de "golpes" que un hombre provoca en la vagina. Este acto es interpretado como el trabajo realizado en el cuerpo de una mujer que el hombre, en este proceso, va a modificar progresivamente.

En Morote y San Juan, los Gitanos se "llevan" a la novia. Es su única forma de matrimonio. Además, a diferencia del caso de la mayoría de los otros Gitanos españoles, este casamiento no implica la desfloración ritual por una Gitana profesional: aquí, los Gitanos no practican la ceremonia del "pañuelo" para averiguar la virginidad de la novia (para los Gitanos de Barcelona, ver Lagunas Arias, 2000a, 2000b y San Román 1976; para los de Madrid, véase Gay y Blasco 1999, 1997; para los de Jerez, ver Caterina Pasqualino, 1998, y para los de Granada, ver Manrique, 2008 y Gamella, 1996). De esta manera, la fuerza y el valor del joven se pone de relieve, pero sobre todo su conocimiento de la red que interpreto como el control de la misma: en este caso, de la moralidad de la joven que, para que un chico se la "lleve", ha de ser, en la forma ideal, virgen. Así, los chicos han de investigar dentro de su red (por lo general con la participación de sus familiares) a propósito de una chica que les gusta en particular. A veces, chicas que "ya están abiertas" consiguen casarse. Pero como dijo Lucas, "el que se lleva a una chica que ya no es virgen no puede quejarse porque él ya lo sabía”. Entonces, es difícil para un hombre quejarse:

14. Malinowski (1922) y Mauss (1925) también sugerían que las relaciones sexuales tienen que ver con el don aunque su percepción del don (puro o no) son diferentes. De hecho, a diferencia de Malinowski, para Mauss, un 'don puro' -"es decir, una ofrenda para la que nada se da a cambio' (Malinowski, 1922: 176)- no puede ser invocado para las relaciones sexuales dentro de una pareja (Mauss, 1925: 172).

15. Cuando una mujer se queda embarazada, las Gitanas dicen "tiene la panza llena". Pero esta expresión se utiliza también para referirse a una mujer que acaba de tener relaciones sexuales: el esperma nutre el cuerpo de la mujer (para más información, ver Manrique 2004). 
esto podría demostrar su falta de conocimiento de las redes locales.

Las mujeres son conscientes de estas categorizaciones basadas en esta relación intrínseca entre el trabajo y el control social. En Morote y San Juan, hacen un punto de honor demostrar su capacidad en trabajar. Por lo tanto, Alicia, una chica de dieciocho años, fulminó un día contra su amiga Piedad, que fue totalmente desconcertada. Resultó que el hermano de Piedad, un Gitano de Asturias encarcelado, había escrito una carta a Alicia en el que declaró su pasión por ella y en que describe la forma en que quería encontrar un trabajo bien remunerado con el fin de evitar que ella trabaje. " $¿ Q u i e ́ n$ se cree que es?" protestó ella: "yo quiero trabajar. No voy a permanecer inactiva mientras que él está trabajando". También son ellas que mayoritariamente se informan sobre la moralidad de una chica. Así que el conocimiento de la red depende del trabajo de las mujeres.

Esta percepción de la actividad femenina no es obviamente compartida por todos los Gitanos españoles. En San Juan y Morote, las mujeres deben demostrar su actividad por su dinamismo y por la aceptación sin pestañear de las tareas más arduas (lo que en realidad cumplen $)^{16}$, mientras que los hombres, a menudo definidos por su carácter activo, no están obligados a justificar de lo que son (ver también Lagunas Arias, 2000a), excepto en los casos de discapacidad (por ejemplo en el caso del abuelo de Rubén).

Las relaciones entre los Gitanos y Castellanos también se integran en el contexto de esta clasificación "natural". Estos últimos son por nacimiento "recipientes" ${ }^{17}$ con respecto a los Gitanos, a pesar de su capital económico a menudo mayor ${ }^{18}$. De hecho, los Payos están clasificados de forma espontánea entre los que están bajo la acción (es decir, que se clasifican como seres pasivos). Al no dar (o poco) y al tratar de maximizar sus bienes a través de la acumulación, parecen reforzar la percepción gitana de su posición fundamentalmente receptiva.

16. Las personas que demuestren mucha voluntad y coraje para enfrentarse a una tarea difícil pueden ser excusadas (no hay burlas) cuando consiguen de manera incorrecta cumplir la tarea.

17. Aunque los Gitanos consideran a los Payos como siendo siempre sus recipientes, controlan el nivel de generosidad que ofrecen. Por ejemplo, recuerdo que al principio de mi estancia en estos dos pueblos, cuando visitaba a una familia gitana siempre había una mujer gitana o niña para preguntarme lo que esta familia me había ofrecido para comer. Después de mi respuesta, si era una mujer, casi siempre me daba su opinión, abiertamente o de forma más discreta, sobre la generosidad de mi anfitriona. Además, esto daba lugar a conversaciones más o menos agitadas en el barrio para evaluar el nivel de generosidad hacia mí. Después de un tiempo, una de mi anfitriona me dió la lista de hombres o mujeres que fueron poco generosos conmigo : "él / ella es una buena persona, él/ella es generoso/a" o "él/ella no es una buena persona en absoluto", con una cara que muestra su desaprobación. Entonces decía: "mejor que nunca vaya a su casa de nuevo", u otras palabras que me demostraban que no eran buenos Gitanos.

18. Los Gitanos son conscientes de esta situación y tratan de sacar el máximo provecho de sus relaciones con el Payo. Esto es lo que Piasere (1984: 139-146) llama el "capital gağikano". 
La generosidad, que implica la distribución de los bienes, el gasto de tiempo o la realización de trabajos en beneficio de personas o grupos de gitanos, es altamente valorizada ${ }^{19}$ y se utiliza para subrayar el bajo estatus del Payo. Así, como lo señala Caterina Pasqualino (1998: 32), "el ideal gitano es vivir en un mundo de verdad, donde se prohíbe el dinero" (mi traducción), donde solo la generosidad crea vínculos entre los individuos. Y añade: "Paco, quien regresó a su casa sin pagar por las bebidas de los demás, se consideró como un tacaño. Fue apodado el Gaché, una expresión que, al igual que el Payo, designa un no Gitano (ibid.: 35)". Del mismo modo, Nancy Thède (2000: 177) explica: "Un valor subyace y orienta los comportamientos identitarios de los Gitanos y los pone, según ellos, en una posición de superioridad sobre los Payos, y este valor es la generosidad" (mi traducción). ${ }^{20}$

Por lo tanto, la circulación continua de bienes (materiales o simbólicos), percibida como generosidad, es el signo de una gitanidad completa. Pero, ¿qué es lo que pasa en las situaciones donde los Gitanos adquieren algo por parte de miembros de categorías inferiores? Voy a argumentar que, a través de un acto que llamo "la toma" (en el sentido de que el donatario no tiene conocimiento de la acción y que por lo tanto no puede aprobar lo que ocurre), los Gitanos que ocupan las categorías superiores transmutan su posición de recipientes en la de tomadores que, por su carácter dinámico, se equivale a la de donantes.

\section{CuAndo TOMAR NO ES ROBAR}

Los Payos están a menudo en una mejor situación económica que los Gitanos. No obstante, los Gitanos les consideran como sus recipientes. De hecho, esta negación de la potencial condición de donatario del Gitano se realiza bajo una puesta en equivalencia entre la posición de donante y la de tomador por la transmutación de lo "naturalmente" pasivo del acto de recibir (la posición paya) en el acto voluntario y activo de la toma. ${ }^{21}$ Entonces, por la toma (que implica trabajo) y a través de la valorización inmoderada

19. Para los Xoraxané situados en Verona (Italia), "existe la acumulación de bienes solo con una función de distribución. Aunque las formas sean diferentes (celebración de nacimiento, del primer corte de pelo, el matrimonio, las ofertas diarias de comida y bebida a los amigos, etc.) los objetivos de distribución son aumentar el prestigio de la cabeza de la familia y para preservar el consenso de los subalternos" (Piasere 1987: 118, nota 4).

20. Una vez, un Castellano de Morote, bastante sorprendido, me explicó que era muy respetada por los Gitanos de este pueblo: me dijo que un día, cuando yo estaba caminando en una calle de la ciudad, oyó a una mujer gitana decir que yo era una persona muy respetable porque había ayudado a varios Gitanos del pueblo y, sobre todo, que era muy generosa al pasar tanto con ellos para ayudarles. Estaba muy sorprendida porque, excepto mis amigos gitanos, nunca sentí que los Gitanos apreciaban mi trabajo.

21. Pienso que esta analogía entre los actos de recepción y de toma puede ser asimilada a la noción de recogida (gathering) que Piasere (1987: 113) utiliza para definir las actividades xoraxané asociadas a la mendicidad y toma (robo): en situación de interacción, dar y recoger son actitudes activas frente a la pasividad funcional de la otra parte de la relación.. 
del dinamismo gitano en contraste con la pasividad "natural" de los Castellanos, la posición subordinada de recipiente de los Gitanos se vuelve, en la jerarquía social gitana, equivalente a la de los donantes.

Para resumir, en la mente gitana, el Gitano no recibe pero coge. Tomar y dar pone el iniciador de la relación en una posición equivalente. Por lo tanto, los Payos no pueden, bajo ninguna circunstancia, ocupar la posición de donante con respecto a los Gitanos. Mientras que algunos (los Gitanos) constantemente renuevan su morfología social y garantizan la igualdad (potencial) entre pares, otros (los Payos) conservan las prerrogativas de sus antepasados se esfuerzan en crear y perpetuar las diferencias de estatus a través de la acumulación de riqueza. Ya que puede causar un desequilibrio de las leyes "naturales" dentro de cada categoría, la riqueza no es deseable para los Gitanos. Las categorías "naturales" basadas en el concepto de generosidad legitiman esta jerarquía, permitiendo que los Gitanos puedan actuar en un mundo payo manteniendo su superioridad autodefinida e integridad gitana (ver más arriba). Por lo tanto, la superioridad gitana está garantizada, independientemente de las relaciones que mantengan con los no Gitanos y con independencia de su posición en la situación interactiva que tengan con los Castellanos.

La toma no solo impide que los Gitanos se categoricen como "recipientes", pero también es para ellos un acto de transformación que pone las relaciones en el orden correcto. Por ejemplo, en una hermosa tarde soleada, mientras caminaba junto a un campo de girasoles con Remedios, Elena, Dulce, Encarna y María, cinco chicas gitanas de Morote, de repente Remedios se precipitó en la plantación, inmediatamente seguida por otras tres chicas. María se extrañó de mi reticencia y me dijo que solían tomar solo un girasol cada una para saborear las pipas. Tomando nota de que todavía quedaba muy reticente, me explicó muy pacientemente que el dueño era muy rico, que su plantación era muy grande, y entonces que era normal tomar algunas flores, con la condición de que la cantidad de flores no supere la cantidad que consideraban equitativa. Me explicó que de alguna manera era la obligación de este hombre distribuir estas cantidades excesivas, demasiado importantes para su propio consumo (de hecho, la toma es, en este caso, de alguna manera una forma de evitar el almacenamiento de bienes y obliga al propietario a ser, a pesar de sí mismo, generoso también). ${ }^{22}$ Con sus comentarios, me parece que María expresaba la idea de que el acto de la toma es, de alguna manera, una forma de poner las cosas en orden. Sentí que las chicas se reían amablemente de mi ignorancia. María también me había comentado unos días antes que había criticado duramente a Manuel porque lo habían pillado varias veces tratando de robar grandes cantidades de almendras (demasiado para tan solo su propia familia) de otro agricultor payo. Concluyó sentencia: "es un ladrón". Para los Gitanos de Morote y San Juan, son los Castellanos 
los que son más bien los ladrones porque, al no permitir la circulación de bienes, y conservándolos para su propio enriquecimiento y empoderamiento, desposeen los Gitanos de su prerrogativa: hacer circular los bienes. En resumen, de acuerdo con las clasificaciones gitanas "naturales", los Gitanos son superiores a los Payos. Como veremos a continuación, con esta misma lógica de circulación de bienes, los hombres también se consideran como superiores a las mujeres.

\section{4. ¿Y LAS MUJERES?}

Las mujeres gitanas parecen a primera vista tener un papel tan importante como los hombres en su comunidad: al igual que sus padres, hermanos, esposos e hijos, trabajan en el campo y contribuyen a la economía familiar, algunas ancianas son muy respetadas, son depositarias del dinero familiar, etc. En realidad, las mujeres no tienen ningún impacto sobre la jerarquía ya que no tienen acceso a lo que podría permitirles capitalizar prestigio simbólico dentro de la comunidad gitana. Es como si las mujeres, que de otro modo hubieran podido competir con los hombres, hubieran estado excluidas desde su nacimiento de cualquier transacción social significativa a nivel comunitario (transmisión de apodos ${ }^{23}$, de sangre ${ }^{24}$, circulación de bienes y alimentos prestigiosos ${ }^{25}$ ). Así que pueden ser donantes pero solo de sus pares (mujeres de su categoría: solteras, casadas o viudas): no pueden perturbar la jerarquía global hecha por y para los hombres.

\section{ENTRe Pares No hay Jerarquía PERENNe}

Dentro de cada categoría (ver figura 1), se supone que debería reinar la igualdad. Este es el resultado del efecto del contradon, que es aquí concebible pero no obligatorio. Entonces, cada uno tiene la capacidad de acumular capital social mediante la demostración de su generosidad: la persona más rica simbólicamente, socialmente hablando, es la que da sus bienes. Pero, ya que cualquier don coloca al recipiente en una posición de subordinación, no existe igualdad absoluta entre pares. Es transitoria y performativa: depende de las nuevas transacciones dentro de la categoría. En este sentido, no se gana nada: en cada generación, cada uno tiene que crear un poco de riqueza (siempre con esfuerzo) con el fin de estar en capacitad de redistribuir. Por lo tanto, no solo los conceptos de trabajo y dinamismo son de gran valor en las transacciones, pero lo más importante, cada hombre debe aumentar sus oportunidades de dar (y no para recibir), lo que le permitirá ampliar su capital social para le conceda un estatus superior entre sus pares. De esta manera,

23. Ver Manrique, 2013

24. Ver Manrique, 2004 y 2009

25. Por ejemplo, los caballos y los canarios y, para la alimentación, las liebres. Para más información, ver Manrique 2008. 
el don reparte y reequilibra las riquezas entre pares con el efecto. Esto conduce a una igualdad temporal de estatus entre pares ${ }^{26}$.

\section{Controlar los lazos SOCiales}

Para los Gitanos de Morote y San Juan, el don es la principal forma de control de las situaciones de interacción mediante el hecho de iniciar toda interacción ${ }^{27}$ : dar es iniciar o renovar las relaciones o legitimar los estatutos implicados. De hecho, hay varias maneras para evitar estar en deuda (o sea de convertirse en un "recipiente") cuando se transfieren bienes a una categoría inferior: por la circulación monetaria (como la compra y venta con $\operatorname{Payos}^{28}$ ) y, como hemos visto, por el robo o toma (según la forma de pensar, gitana o no, del locutor). Sin embargo, entre pares estos mecanismos están muy mal visto. Entonces aunque no sea deseable, el don es la única forma de relación aceptable entre Gitanos de misma categoría. Además, es la única manera de crear continuamente o actualizar situaciones de interacción que pueden dar lugar a nuevas alianzas, y así dar la capacidad a cada individuo de manejar la morfología de las redes en las que está inmerso: el que da -es decir, el que inicia el vínculo social asigna (temporalmente) la posición de recipientes a los destinatarios del don.

Podríamos preguntarnos el porqué de esta búsqueda constante de control. De hecho, ya que estas personas son una minoría, el poder y control a nivel global que son capaces de ganar solo puede ser muy restringido. En realidad, para tener el poder y/o autoridad en la sociedad general, sería mejor para ellos adoptar los hábitos de comportamiento de otras personas españolas (como la transmisión de la riqueza, la aspiración a una mejor educación, la búsqueda de una carrera política o religiosa, etc.). Pero a pesar de una política de discriminación positiva hacia ellos en toda la región andaluza desde hace muchos años, e incluso aunque otros gitanos españoles han avanzado en esta dirección, los Gitanos de Morote y San Juan no lo hacen (en 1998, casi el 100 por ciento de los gitanos de más de veinte años de edad abandonaron la escuela antes de la edad de dieciséis años,

\footnotetext{
26. Este concepto de riqueza parece ser extendido en el mundo gitano y rom. Por lo tanto, un proverbio Romani dice que "Si no tienes nada, los Rom te darán, si tienes mucho, los Rom tomarán de ti" (Courthiade y Meritxell, 2006: 37).

27. Podemos hacer aquí una comparación con algunos Roma húngaros que compran y venden caballos que comercian con campesinos no gitanos (ver Stewart, 1992). De hecho, según el antropólogo (ibíd.: 107): "En este juego (el mercado)... me parece que es el fin de disfrutar del placer de la creación de un acuerdo, más que por tener alguna remuneración, que los Gitanos juegan el papel de Cincár (es decir, de intermediario). Esto le hace ser un 'verdadero Gitano"'(108 ibíd.).

Poco dinero circula de manera directa entre Gitanos (generalmente son préstamos). Así me contó una Gitana de Asturias que un Gitano de San Juan quiso venderle canastas. Cuando ella le dijo que era gitana, se disculpó por el error y se fue rápidamente.
}

28. Si no es una cueva, toda la familia se muda a otra casa sin quemarla. Pero solo los Payos aceptarían vivir en ella. 
y casi el 99 por ciento de los más de cuarenta años eran analfabetos algunos, solo sabían firmar).

Según Graeber (2011: 51), en la realeza sagrada, el control del cuerpo es de primordial importancia: "En la medida en que el Reth es la encarnación de la nación y de la humanidad en su conjunto frente a los poderes divinos, en la medida en que es el ser humano genérico, él debe ser el hombre perfecto".

Entonces, puede que los Gitanos traten de controlarlo todo con el fin de evitar la pérdida de una parte de su gitanidad (lo que en otras palabras, significa tratar de ser perfecto), pero hay una cosa que nunca podrán superar: su devenir después de la muerte. ¿Cómo, entonces, hacer frente a este problema sin solución?

\section{LA RIQUEZA NO SE PUEDE HEREDAR}

El hecho de no acumular riqueza se hace más evidente en el caso de la muerte de un individuo. Después de la muerte de un Gitano de San Juan o Morote, la transmisión de sus bienes es impensable (ver Williams, 1993 para una descripción similar entre los Manuches). De hecho, parece que cuando alguien muere, es como si la mecánica del don pudiera causar la muerte de los relativos consanguíneos o aliados de los fallecidos. En efecto, cuando una muerte está a punto de suceder, gran agitación sacude a toda la comunidad: todos los Gitanos que tienen o han tenido cualquier tipo de enlace (sea de parentesco, de matrimonio o de comercio, etc.) con la persona que se está muriendo, se precipitan, a veces cumpliendo varios cientos de kilómetros de distancia, con el fin de "demostrar su respeto" y, en mis palabras, para deshacer cualquier compromiso que tenían con el moribundo antes de su fallecimiento.

En realidad, se hace todo posible para romper cualquier vínculo (de cualquier forma) con el fallecido y privarlo de cualquier función en las redes de intercambio del don. Para confirmar esta ruptura con la sociedad de los vivos (se cree que el individuo muerto puede permanecer entre los vivos o volver del mundo de los muertos), no se pueden conservar los bienes asociados a los difuntos que se queman o se entierran (sus pertenencias personales, como su ropa, su cuchillo, su bastón, su moto, y su casa, si se trata de una cueva $^{29}$ y oficialmente se "olvidan" (como los apodos por ejemplo: ver Manrique, 2008). De esta manera, la jerarquía que supervisa toda la organización social gitana, es decir, la clasificación en categorías "naturales", así como la igualdad entre pares establecidas en el nacimiento, se mantienen: categorías/personas de niveles bajos no pueden acumular los bienes adquiridos por las generaciones anteriores. Por lo tanto, los estatutos de los Gitanos, que no se transmiten por herencia de riquezas pero solo por el concepto del don (ya sean bienes materiales o simbólicos), no son transmisibles.

29. Si no es una cueva, toda la familia se muda a otra casa sin quemarla. Pero sólo los Payos aceptarían vivir en ella. 
Para resumir, los Gitanos de San Juan y Morote distinguen los seres vivos de acuerdo con el principio de la entrega de regalos, en la que algunos están integrados en el mecanismo de intercambio (con el derecho y debe de dar), mientras que otros se encuentran fuera de las reglas de la comunidad; esta dinámica en realidad solo se refiere a los seres humanos ya que los animales y las plantas no pueden ser donantes (Manrique, 2008: 151). Las categorías, cuanto más alejadas de la parte superior, más diferenciadas son de la completitud y de naturaleza humana. Ser rico es entonces como un síntoma de "salvajismo", lo que es lo contrario del ideal gitano, porque revela la propia renuencia a dar y la preferencia de uno para la acumulación de riquezas. Así que, incluso si la muerte siempre se ha sentido como un acontecimiento terriblemente dramático, es en otro sentido, la posibilidad para la comunidad de hacer circular o destruir todos aquellos bienes que han sido acumulados por una persona durante su vida. En la percepción gitana de los vínculos sociales, la muerte podría representar el punto de vista de la anomia -es decir, la posible pérdida de control del campo social de los vivos si los lazos perduran con el fallecido o la fallecida (los muertos podrían hacer lo que quieren, por ejemplo, reaparecer) y así la pérdida de parte de su identidad gitana. En estas circunstancias, es mejor cortar toda relación con los muertos. 


\section{REFERENCIAS BIBLIOGRÁFICAS}

Ardévol Piera, Elisenda (1986) "Vigencias y cambio en la cultura de los gitanos", en Teresa San Román (coord.), Entre la marginación y el racismo. Reflexiones sobre la vida de los gitanos. Madrid: Alianza editorial, pp. 61-108.

Augustins, Georges (1986) “La perpétuation des Groupes Domestiques dans les Sociétés Paysannes Européennes”. Tesis doctoral. Universidad de Paris X Nanterre.

Bestard Camps, Joan (1986) Casa y familia. Parentesco y reproducción doméstica en Formentera. Palma de Mallorca: Institut d'Estudis Baleàrics.

Bourdieu, Pierre (1980) "Le capital social. Notes provisoires". Actes de la Recherche en Sciences Sociales, 31(31), pp. 2-3.

Cervantes Saavedra, Miguel (de) (1990). La gitanilla. Barcelona: Ediciones 29, (1 $1^{\text {era }} \mathrm{ed}$. 1613).

Courthiade, Marcel (2004) "Kannaus on the Ganges, cradle of the Rromani people". En Donald Kenrick (Coord.) Gypsies: from the Ganges to the Thames. Hertfordshire, Great Britain: University of Hertfordshire Press, Collection Interface, pp. 105-126.

Courthiade, Marcel y Meritxell, Stella (2006) Sagesse et humour du peuple rrom. Proverbes bilingues rromani-français. Paris: L’Harmattan.

Descola, Philippe (2011) Par-delà nature et culture. Paris: Editions Gallimard, nrf, (1 era $^{\text {en }}$ ed. 2005).

Gay y Blasco, Paloma (1997) "A 'Different Body'? Desire and Virginity among the Gitanos". The Journal of the Royal Anthropological Institute, n.s.(3). London: pp. 517-535.

Gay y Blasco, Paloma (1999) Gypsies in Madrid. Sex, Gender and the Performance of identity. Oxford \& New-York: BERG.

Graeber, David (2010) "Les fondements moraux des relations économiques. Une approche maussienne". Revue du Mauss, 36, pp. 51-70.

Graeber, David (2011) "The divine kingship of the Shilluk. On violence, utopia, and the human condition, or, elements for an archaeology of sovereignty". Hau: Journal of Ethnographic Theory, 1(1), pp. 1-62.

Gropper, Rena Cotten (1975) Gypsies in the city. Culture patterns and survival. Princeton, New Jersey: The Darwin Press.

Hertz, Robert (1990) "la preeminencia de la mano derecha". En La Muerte y la mano derecha. Madrid. Alianza Editorial, pp. [1 ${ }^{\text {era }}$ ed. 1928].

Lagunas Arias, David (2000a) “Dentro de 'dentro': Estudio antropológico y social de una comunidad de gitanos catalanes”. Tesis doctoral. Jaén: Universidad de Jaén. 
Lagunas Arias, David (2000b) "Rethinking Gitano Kinship. The Calós of Catalonia", Europcea, VI(1/2), pp. 159-193.

Leblon, Bernard (1985) Les Gitans d'Espagne. Paris: Presses Universitaires de France.

Lévi-Strauss, Claude (1949) Les Structures élémentaires de la parenté. Paris: Presses Universitaires de France.

Malinowski, Bronislaw (1922) Argonauts of the Western Pacific: An Account of native enterprise and adventure in the archipelagos of Melanesian New Guinea. London: Routledge.

Manrique, Nathalie (2004) "La lune pétrifiée. Représentations parthénogénétiques dans une communauté gitane (Grenade)”. En Françoise Héritier y Margarita Xanthakou (Coords) Corps et Affects. Paris: Odile Jacob, pp. 205-220.

Manrique, Nathalie (2008) “'Sois généreux'! Du don comme principe structurant de l'organisation sociale des Gitans de deux petits bourgs andalous (Espagne)". Tesis doctoral. Paris: Ecole des hautes études en sciences sociales.

Manrique, Nathalie (2009) "Corpo-real identities: Perspectives from a Gypsy Community". En Jeanette Edwards y Carles Salazar (Coords) European Kinship in the Age of Biotechnology. Oxford \& Manchester: Berghahn Books, pp. 97-111.

Manrique, Nathalie (2010) "La moustache de la distinction". En Dimitri Karadimas (coord.), Cahiers d'anthropologie sociale, 6, Éditions de L’Herne, pp. 85-93.

Manrique, Nathalie (2013) "Vocabulaire de parenté gitan: une terminologie marquée par le don”. L'Homme, pp. 35-54.

Manrique, Nathalie. (próxima publicación) “Sois généreux!'. Don et hiérarchie chez les Gitans de deux petits bourgs andalous (Espagne)". Paris: Petra.

Mauss, Marcel (1925) "Essai sur le don: Forme et raison de léchange dans les sociétés archaïques”, Paris: L'Année Sociologique, 1, pp. 30-186.

McCallum, Cecilia (2009) Gender and sociality in Amazonia: How real people are made, New-York \& Oxford: Berg.

Okely, Judith (1983) The Travellers-Gypsies. Cambridge: Cambridge University Press.

Pasqualino, Caterina (1998) Dire le chant. Les Gitans flamencos d'Andalousie. Paris: CNRS Éditions \& Éditions de la Maison des Sciences de L’Homme.

Piasere, Leonardo (1984) "Mare Roma. Catégories humaines et structure sociale. Une contribution à l'ethnologie tsigane". Tesis doctoral. Paris: École des Hautes Études en Sciences Sociales. 
Piasere, Leonardo (1987) "In Search of New Niches: The Productive Organization of the Peripatetic Xoraxané in Italy”. En Aparna Rao (Coord.) The Other Nomads. Cologne: Böhlau, pp. 111-132.

San Román, Teresa (1976) Vecinos gitanos. Madrid: Akal Editor.

Sánchez Ortega, Maria Helena (1977) Documentación selecta sobre la situación de los Gitanos Españoles en el siglo XVIII. Madrid: Biblioteca de visionarios, Editora Nacional. Segalen, Martine (1985) Quinze Générations de Bas-Bretons. Parenté et société dans le pays bigouden sud. 1720-1980, Paris: Presses Universitaires de France.

Stewart, Michael (1992) "Gypsies at the Horse-Fair". En Roy Dilley (Coord.) Contesting markets. Analyses of Ideology, Discourse and Practice. Edinburgh: Edinburgh University Press.

Stewart, Michael (1994) "La passion de l’argent: Les ambiguités de la circulation monétaire chez les Tsiganes hongrois”. Terrain: Les usages de l'argent, 23, pp. 45-62.

Testart, Alain (2013) “What is a gift?”. Hau: Journal of Ethnographic Theory, 3, 1, pp. 249261 (translated from Chapter One of Critique du don. 2007).

Thède, Nancy (2000) Gitans et Flamenco. Les rythmes de l'identité. Paris \& Montréal: L'Harmattan.

Viveiros de Castro, Eduardo (2009) Métaphysiques cannibales: Lignes d'anthropologie post-structurale. Paris: Presses Universitaires de France (translation by Oiara Bonilla).

Williams, Patrick (1993) Nous, on nien parle pas: les vivants et les morts chez les Manouches. Paris: Editions de la Maison des Sciences de l'Homme.

Zonabend, Françoise (1980) La mémoire longue. Temps et histoires au village. Paris: Presses Universitaires de France. 